\title{
A Novel Phenomenological Multi-physics Model of Li-ion Battery Cells
}

\author{
Ki-Yong Oh, Nassim A. Samad, Youngki Kim, Jason B. Siegel, Anna G. Stefanopoulou, and
}

Bogdan I. Epureanu*

Department of Mechanical Engineering, University of Michigan, 2350 Hayward Street, Ann

Arbor, MI 48109-2125,USA

*Corresponding author: Tel.: +1-734-647-6391; fax: +1-734-615-6647

E-mail address: epureanu@umich.edu

\begin{abstract}
A novel phenomenological multi-physics model of Lithium-ion battery cells is developed for control and state estimation purposes. The model can capture electrical, thermal, and mechanical behaviors of battery cells under constrained conditions, e.g., battery pack conditions. Specifically, the proposed model predicts the core and surface temperatures and reaction force induced from the volume change of battery cells because of electrochemically- and thermally-induced swelling. Moreover, the model incorporates the influences of changes in preload and ambient temperature on the force considering severe environmental conditions electrified vehicles face. Intensive experimental validation demonstrates that the proposed multi-physics model accurately predicts the surface temperature and reaction force for a wide operational range of preload and ambient temperature. This high fidelity model can be useful for more accurate and robust state of charge estimation considering the complex dynamic behaviors of the battery cell. Furthermore, the
\end{abstract}


inherent simplicity of the mechanical measurements offers distinct advantages to improve the existing power and thermal management strategies for battery management.

\section{Keywords}

Battery management system; Li-ion battery; Li-ion intercalation induced stress and strain; Multiphysics model; Thermal stress and strain

\section{Introduction}

Rechargeable Li-ion batteries (LIBs) have various advantages compared to alternative batteries. LIBs not only provide high power/energy density over a broad temperature range of operation, but also exhibit no memory effect, low self-discharge ratio, and long cycle life. [1-4]. These advantages make LIBs an ideal candidate for a wide variety of applications from small-scale portable electronics to massive-scale energy storage systems.

However, problems that persist in existing LIBs limit their application in transportation, military, and aerospace due to the stringent safety standards. The limitations of current battery technology include underutilization, capacity fade, thermal runaway, and stress-induced material damage. In order to overcome these challenges, understanding the complex multi-physics beyond the LIBs is indispensable.

The significant efforts have been devoted to identify the complex physics behind the LIB which would be useful to predict operational states, and thereby enhances operational safety and 
enlarges operational window. The porous electrode theory, which solves Lithium diffusion dynamics and charge transfer kinetics in a paired intercalation electrode system, has been proposed to predict the electrical response of a cell [6]. This physics-based model can predict microscopic behavior and performance, whereas it requires a large computational power to solve the differential equations. An equivalent circuit model has also been proposed for controloriented purposes to estimate the electrical response and the amount of heat generation [7-9]. A variety of heat transfer models have been created and validated through experiments [10-13]. Several lumped parametric thermal models have also been proposed for control-oriented purposes with advanced power management schemes [14-16]. Numerical simulations on the cell-level and pack-level with computational fluid dynamics and finite element methods have been conducted to predict the thermodynamics of Li-ion battery cells and packs with experimental validation [17-20]. Coupled models between electrochemistry and heat transfer have also been suggested to elucidate the coupled effect of the current, potential, and temperature on the state of charge (SOC) and state of health (SOH) for large-scale LIBs [21-24].

Recent research focuses more on the structural response. The volume change of electrode materials in LIBs under charge process has been intensively investigated to characterize the electrochemical-induced stress and strain [25-32]. The effects of prestress and stress-evolution on capacity fade over time or cycling have also been studied [33,34]. This research promotes theoretical and experimental understanding of the structural response of the LIBS, especially in microscopic perspective. Moreover, the macroscopic stress and strain responses from two sources, i.e. Li-ion intercalation and temperature variation, is observable and measureable with the advancement of sensor technology, suggesting that the structural response can become a sensitive gauge for characterizing the battery state [5,35]. However, studies related to stress and 
strain on the cell-level are still few, especially in modeling perspective. Moreover, the coupled model of stress and strain with electrochemical reaction and thermodynamics of the LIBs has not been investigated in great detail, even though this fully-coupled multi-physics model can improve the safety and reliability of batteries, enhance the capability of cells and packs, and eventually prolong the lifetime of the LIBs.

This paper proposes a fully-coupled phenomenological multi-physics model of the LIBs for the first time. The proposed multi-physics model couples the electric, thermal, and swelling effect on the force in pack conditions. The important parameters, which govern the thermal characteristics and mechanical responses on the cell-level, were estimated from experimental data. The main purpose of this phenomenological model is to predict the temperature and force induced from the volume change of battery cells, which is driven by prestress, Li-ion intercalation, and temperature variation, in pack conditions. Experimental validation at a variety of operational conditions confirms that the proposed multi-physics model accurately predicts the temperature and the compression force during operation at the overall SOC regions for a wide range of temperature and preload conditions.

\section{Experiments}

This study used a flat-wound type prismatic $5 \mathrm{Ah} \mathrm{Li-ion} \mathrm{cell} \mathrm{obtained} \mathrm{from} \mathrm{a} \mathrm{Ford} \mathrm{Fusion} \mathrm{HEV}$ battery pack. Detailed information of the Li-ion cell is available in Ref. [5].

Three LIBs were sandwiched together between two Garolite end-plates and bolted to maintain a constant compression length to replicate conditions experienced in a battery pack. The battery 
cells were separated by spacers that maintain compression between the cells while allowing for airflow between them for cooling purposes (Figure 1). Battery temperature was measured on the surface of the cell by using resistance temperature detector sensors (RTDs). Battery force was measured by using four load cells, Omega LC8150-250-100 sensors with a $450 \mathrm{~N}$ full-scale range and an accuracy of $2 \mathrm{~N}$, placed on the corners of the fixture. The fixture was placed in a thermal chamber for ambient temperature control, and the force and temperature data were collected via an 18-bit data acquisition card and a National Instruments module. Bitrode model FTV was used for battery cycling.

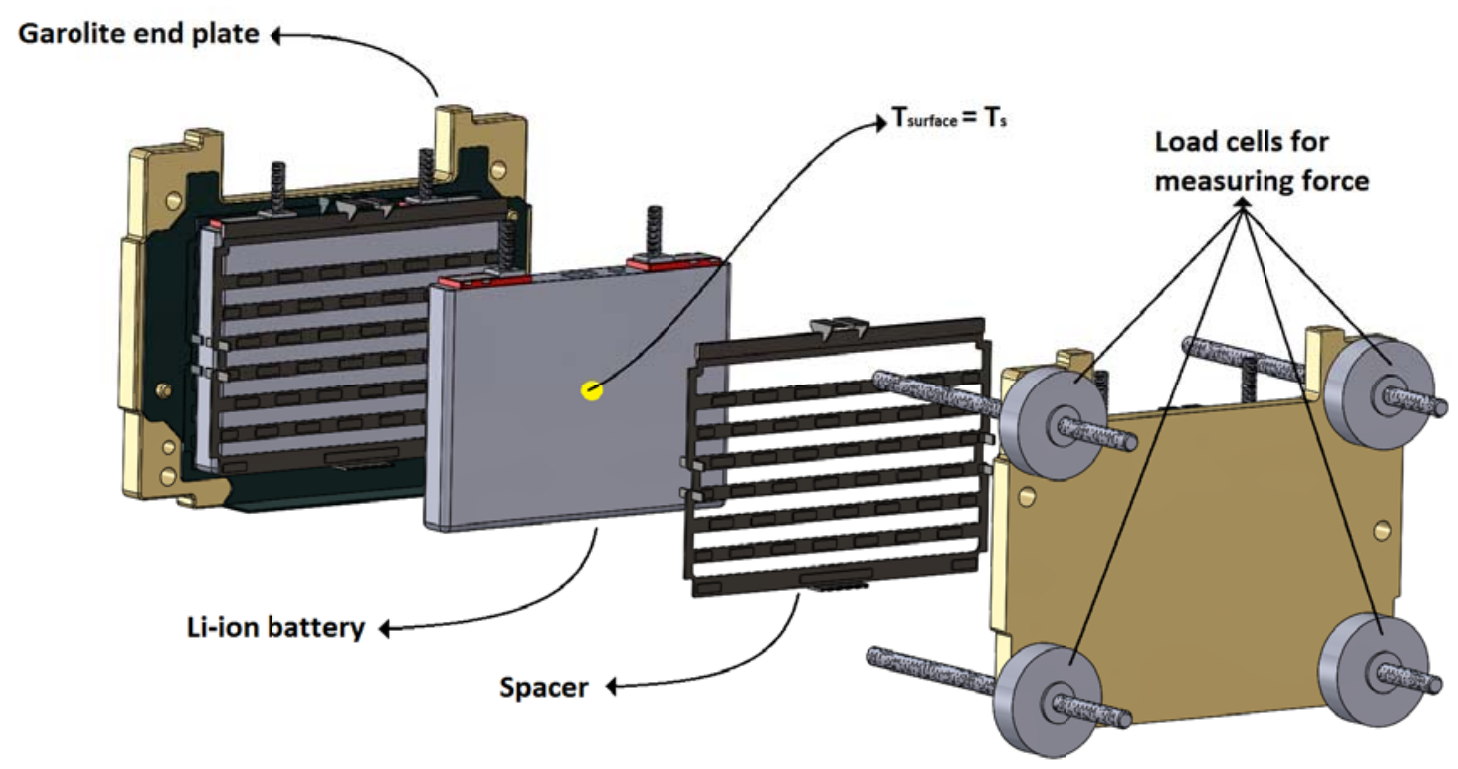

Figure 1. Schematic diagram of the experimental setup showing the fixture, the cell, the spacer, and the sensor location.

Several experiments were performed to characterize and validate parameters used in the multiphysics model and are outlined below. 
In the first experimental sets, the quasi-static force was measured at several different ambient temperature and two different preload conditions. Prior to discharge, the battery was fully charged using a CCCV protocol [35] at $5 \mathrm{~A}(1.0 \mathrm{C})$ and rested three hours at a fixed ambient temperature of $25{ }^{\circ} \mathrm{C}$ as regulated by the thermal chamber; the voltage was clamped after reaching 4.1 V, at which time it was held until the current tapered to $\mathrm{C} / 100(50 \mathrm{~mA})$. Then, the temperature of the thermal chamber was changed to the desired temperature $\left(-5^{\circ} \mathrm{C}, 10{ }^{\circ} \mathrm{C}\right.$, $45^{\circ} \mathrm{C}$ ). Each temperature variation was followed by three hours of rest time to ensure thermal equilibrium. This procedure was omitted to measure the quasi-static force at the ambient temperature of $25{ }^{\circ} \mathrm{C}$. In order to obtain the desired SOC ranging from $0 \%$ to $100 \%$ with $5 \%$ interval, the battery was discharged with a $0.4 \mathrm{C}$ current of actual capacity with an appropriate time ( 7.5 minutes). The actual capacity was calculated by using coulomb counting method during discharge with $0.4 \mathrm{C}$ rate from $4.1 \mathrm{~V}$ to $3.0 \mathrm{~V}$ herein. Each discharge was followed by three hours of rest time to ensure the quasi-static equilibrium. This procedure was repeated four times with the identical preload, $670 \mathrm{~N}$ at $0.05 \mathrm{SOC}$, but different four ambient temperature ($5{ }^{\circ} \mathrm{C}, 10{ }^{\circ} \mathrm{C}, 25^{\circ} \mathrm{C}, 45^{\circ} \mathrm{C}$ ) during discharge. These four experiments were also repeated again with different preload, i.e. $450 \mathrm{~N}$ at $0.05 \mathrm{SOC}$.

In the second experimental sets, three pulse excitation experiments were performed for validating the estimated coefficient of thermal expansion at an ambient temperature of $25^{\circ} \mathrm{C}$ and the wide range of preload conditions. The battery was fully charged using standard CCCV protocol prior to discharge. Then, the battery was discharged with a $2 \mathrm{~A}(0.4 \mathrm{C})$ current for appropriate time to obtain three desired SOC. In the first experiment, a 50 A charge sustaining pulse with a 1 second period ( 0.5 second charge and 0.5 second discharge) was applied for 2.5 hours at 0.48 SOC. The initial preload was set to $1276 \mathrm{~N}$. In the second experiment, a $50 \mathrm{~A}$ 
charge sustaining pulse with a 100 -second period (50 second charge and 50 second discharge) was applied for 2.5 hours at $0.22 \mathrm{SOC}$ with an initial preload of $145 \mathrm{~N}$. The final experiment consisted of a 50 A charge sustaining pulse with a 100 -second period (50 second charge and 50 second discharge). The pulse was applied at 0.74 SOC with an initial preload of $330 \mathrm{~N}$.

In the final experimental sets, intensive experimental validation was performed for the proposed multi-physics model with the US06 duty cycle at a variety of operational conditions (Table 1). A current profile measured from a Ford Fusion hybrid over US06 driving cycle was applied in experimental sets.

Three additional fixtures (Figure 1) were prepared to collect the second and third experimental sets. Acquiring one set of data (shown as symbols in Figures 4 (a) and (b)) takes one week in the first experimental setup. In total, the first experimental setup required two months to collect the data shown in Figure 4. Therefore, conducting experiments in parallel with four fixtures significantly reduced the time needed to gather the experimental data. Moreover, obtaining data with different cells has allowed us to guarantee that the proposed model is adequate for simulating the behavior of different cells. Additional details on the applicability of the model are discussed in Section 4.4.

Table 1. Conditions for the set of US06 duty cycle experiments used for the validation of the multi-physics model.

\begin{tabular}{|c|c|c|c|}
\hline Experiment & Initial SOC (\%) & Ambient Temperature $\left({ }^{\circ} \mathrm{C}\right)$ & Initial Preload $(\mathrm{N})$ \\
\hline 1 & 50 & 25 & 935 \\
\hline 2 & 50 & 11 & 950 \\
\hline 3 & 33 & 11 & 550 \\
\hline 4 & 50 & 11 & 600 \\
\hline 5 & 66 & 11 & 680 \\
\hline
\end{tabular}




\section{Model description}

The multi-physics model consists of three major components (Figure 2): a coupled ElectroThermal Model (ETM), swelling models, and a force estimator. The ETM estimates the state of charge (SOC) and the surface/core temperature of the battery cell with the measured current and ambient temperature. The swelling models calculate the total amount of swelling for the battery cell at unconstrained conditions with SOC and the surface/core/ambient temperatures provided by the ETM. The force estimator calculates the reaction force caused by the volume change of the battery cell from Li-ion intercalation, temperature variation, and preload.

When creating the multi-physics model, the following assumptions are made:

1) The hysteresis of swelling and force during Li-ion intercalation and deintercalation is negligible.

2) The Li-ion intercalation swelling of battery cells in terms of SOC is not affected by temperature in the range of experiments $\left(-5^{\circ} \mathrm{C}\right.$ to $\left.50{ }^{\circ} \mathrm{C}\right)$.

3) The equivalent coefficient of thermal expansion for a battery cell at constrained conditions can be different to that at unconstrained conditions. Gaps/voids can easily generate and thereby mechanical magnification of ruffling can occur at unconstrained conditions [36,37]. Moreover, mechanical constraints also affect the local swelling over surface in constrained conditions $[5,52]$. 
4) The material properties for battery cells can change with respect to SOC because of the phase transition.

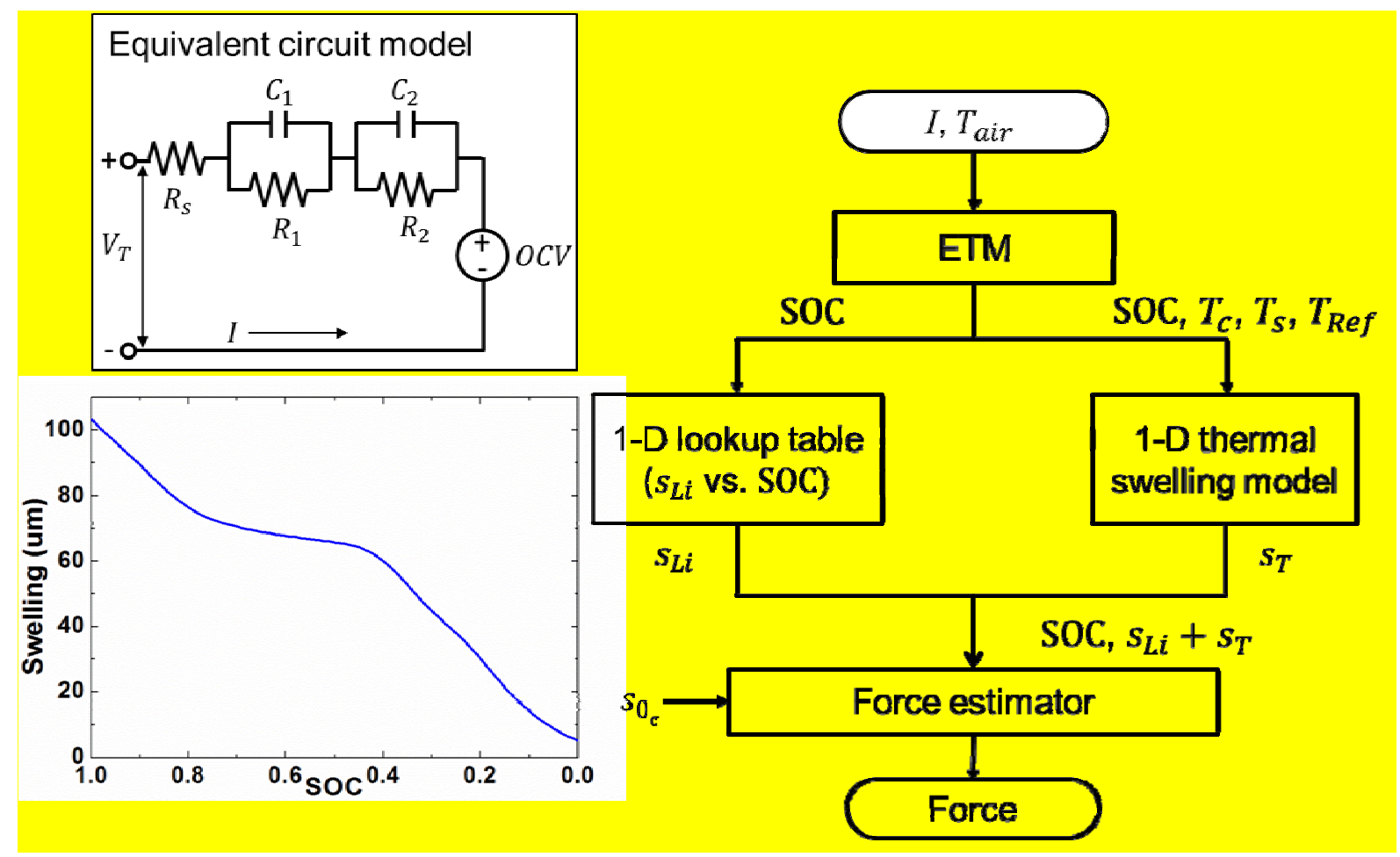

Figure 2. Overall structure of the multi-physics model for LIBs; the inset figure on the left-top side shows an equivalent circuit model; the inset figure on the left-bottom side shows swelling versus SOC measured at $0.2 \mathrm{C}$.

\subsection{Coupled Electro-Thermal Model (ETM)}

A coupled ETM was used to predict electric and thermal dynamics of the battery cell. The ETM is comprised of a three-state electrical model and a two-state thermal model $[38,39]$. 
Electrical dynamics of the battery cell including state-of-charge, polarization voltage, and terminal voltage are predicted with an equivalent circuit model (the inset figure on the top-left side of Figure 2), which describes a cell as one serial resistance and double RC pairs as

$$
\left[\begin{array}{c}
\dot{Z} \\
\dot{V}_{1} \\
\dot{V}_{2}
\end{array}\right]=\left[\begin{array}{ccc}
0 & 0 & 0 \\
0 & \frac{-1}{R_{1}(T, z) C_{1}(T, z)} & 0 \\
0 & 0 & \frac{-1}{R_{2}(T, z) C_{2}(T, z)}
\end{array}\right]\left[\begin{array}{c}
Z \\
V_{1} \\
V_{2}
\end{array}\right]+\left[\begin{array}{c}
-\frac{1}{Q} \\
\frac{1}{C_{1}(T, z)} \\
\frac{1}{C_{2}(T, z)}
\end{array}\right][I]
$$

where $z$ is the battery SOC, $Q$ is the capacity of the cell, $T$ is the temperature, and $I$ is the current; $V_{1}$ and $V_{2}$ are the voltages across resistors $R_{1}$ and $R_{2}$ (or across capacitors $C_{1}$ and $C_{2}$ ) respectively. One serial resistance accounts for battery Ohmic losses including the resistances of current collectors, active material, electrolyte, and separators. $R_{1}$ and $R_{2}$ represent the effective resistance characterizing electrochemical polarization and the effective resistance characterizing the transient concentration polarization, whereas $C_{1}$ and $C_{2}$ are used to characterize the transient response during transfer of power to/from the battery corresponding to the electrochemical polarization and the concentration polarization separately [40]. Eq. (1) above is a state-space representation of the differential equations governing the dynamic electrical response of the cell. The terminal voltage of the cell $\mathrm{V}_{T}$ can be calculated as

$$
\mathrm{V}_{T}=V_{O C V}(z)-V_{1}-V_{2}-I \cdot R_{S}(T, z),
$$

where $R_{s}$ is the series resistance. To solve for the terminal voltage $V_{T}$, the three states, i.e. the state of charge (denoted by $z$ ), $V_{1}$ and $V_{2}$, have to be solved by using Eq. (1) at every time instant. Specifically, given any initial conditions of states $z, V_{1}$, and $V_{2}$, and any current input $I$, the time evolution of the states can be solved using Eq. (1), and then substituted into Eq. (2) to predict the terminal voltage. 
The electrical parameters $R_{s}, R_{1}, R_{2}, C_{1}$ and $C_{2}$ are a function of temperature $T$ and SOC. These values were parameterized by pulsing the cell at different SOCs and temperatures. Specifically, a series of current pulses were applied at different SOCs and temperatures. Using the voltage relaxation data, one can extract the electrical parameters. Ref. [38] provides more details on the parameterization schemes used and on the procedures to identify the electrical parameters.

The heat transfer of the battery cell is captured by a lumped thermal capacitance approach. The battery cell is assumed to have interior and surface nodes with thermal capacitances, and the two nodes are connected through a thermal resistance. Since the cell is thermally represented by two nodes (core and surface), Eqs. (3) and (4) represent the heat transfer between these nodes. The heat generated at the core node is conducted to the surface node and then convected to the ambient air.

The thermal capacitance is determined from the density $\rho$, specific heat capacity $c$, and volume V. The thermal resistance is determined from thermal conductivity $K$, thickness $L$, and area $A$. The heat transfer on the core of the cell is

$$
(\rho \mathrm{cV}){ }_{c} \dot{T}_{c}=\frac{K A}{L}\left(T_{s}-T_{c}\right)+I^{2} R_{S}(T, z)+\frac{V_{1}^{2}}{R_{1}(T, z)}+\frac{V_{2}^{2}}{R_{2}(T, z)}+I T_{c} \frac{d U}{d T}(z),
$$

where $\frac{d U}{d T}$ is the entropy slope. Subscript $c$ and $s$ denote core and surface quantities.

The first term on the right hand side of Eq. (3) represents the heat conduction between the core node and the surface node of the cell, whereas the rest of the terms represent the heat generation.

The term $I^{2} R_{S}(T, z)+\frac{V_{1}^{2}}{R_{1}(T, z)}+\frac{V_{2}^{2}}{R_{2}(T, z)}$ represents Ohmic heat generation, whereas the term $I T_{c} \frac{d U}{d T}(z)$ denotes the entropic heat generation. The amount of heat generated in the jellyroll 
varies upon the battery SOC and temperature $T$ because resistances are a function of temperature and SOC and the entropy slope $\frac{d U}{d T}$ is a function of SOC. The heat transfer on the surface of the cell is

$$
(\rho \mathrm{cV})_{s} \dot{T}_{s}=\frac{K A}{L}\left(T_{c}-T_{S}\right)+h A\left(T_{a m b}-T_{S}\right)
$$

where $T_{a m b}$, and $h$ denote the ambient temperature, and the heat transfer coefficient on the surface of the cell [39]. The first term of the right hand side of Eq. (4) represents the heat conduction between the core node and the surface node of the cell, and the second term is the heat convection to the ambient air. The thermal parameters are constants and were obtained applying a charge sustaining pulsing profile and fitting the thermal data to the thermal model. Ref. [38] provides more details on the characterization of thermal parameters. Note that thermal capacitance for the surface and the core is different in the sense that the core node represents jellyrolls, whereas the surface node represents aluminum casing.

\subsection{Swelling models}

The volume of the LIBs is changed from two sources in unconstrained conditions: Li-ion intercalation and temperature variation. However, this volume change is also affected by the preload in constrained conditions; the preload creates the initial displacement. Therefore, the total swelling from three sources should be considered to accurately predict the corresponsive reaction force during operation. Swelling models predict the volume change of LIBs originated 
from $\mathrm{Li}$-ion intercalation and temperature variation. Initial displacement is estimated from the force estimator in that initial displacement is caused by the preload.

The interlayer spacing of atoms in the active material particles, which make up the electrodes, is affected by Li-ion concentration because the amount of $\mathrm{Li}$-ion in each electrode varies upon lithiation when lithiation and delithiation are occur concurrently in the cathode and anode. Especially, the layered structure such as graphite intercalation compounds shows significant volume change, whereas the spinel and olivine structures such as $\mathrm{LiMn}_{2} \mathrm{O}_{4}$ and $\mathrm{LiFePO}_{4}$ minimally vary upon lithiation [41], suggesting that the volume change of graphite anode mainly contributes to the volume change of battery cells.

The swelling originated from Li-ion intercalation is calculated by using a lookup table. Measured swelling below $0.2 \mathrm{C}$ can be used to establish a data set (e.g. a lookup table) to use when estimating pure Li-ion intercalation swelling. The temperature variation during discharge is below $0.1{ }^{\circ} \mathrm{C}$ when the battery cell is operated below $0.2 \mathrm{C}$; the standard deviation and the average of temperature variation during $0.2 \mathrm{C}$ discharge are $0.05{ }^{\circ} \mathrm{C}$ and $25.51{ }^{\circ} \mathrm{C}$. Hence, data at or below $0.2 \mathrm{C}$ allow direct correlation to be made between swelling and $\mathrm{Li}$-ion intercalation in a cell sandwich, without significant convolution with thermal expansion. The set of data showing swelling versus SOC (inset figure on the left-bottom side of Figure 2) was measured at $0.2 \mathrm{C}$.

Thermal swelling on the cell-level is similar in order of magnitude with Li-ion intercalation swelling, suggesting that thermal swelling is far from insignificant. Therefore, thermal swelling is estimated [42] as

$$
s_{T}(t)=\alpha_{b a t}(T, t) L_{b a t}\left[\frac{2}{3}\left(T_{c}(t)-T_{s}(t)\right)-\left(T_{s}(t)-T_{a m b}\right)\right],
$$


where $s_{T}, \alpha_{b a t}, L_{b a t}, t$ represent the thermal swelling of the cell, the equivalent coefficient of thermal expansion of the cell, the original thickness of the cell, and time. This estimated thermal swelling is added to Li-ion intercalation swelling and initial displacement to calculate the total volume change of the cell in constrained conditions.

This model accounts for the non-uniform temperature distribution throughout the battery cell to accurately predict the thermal swelling. Specifically, the first term, $\frac{2}{3} \alpha_{b a t}(T, t) L_{b a t}\left(T_{c}(t)-\right.$ $\left.T_{S}(t)\right)$, represents the thermal swelling due to temperature variation across the cell. The second term, $\alpha_{b a t}(T, t) L_{b a t}\left(T_{s}(t)-T_{a m b}\right)$, represents the thermal swelling due to the difference between the current surface temperature and the ambient temperature. Note that the equivalent coefficient of thermal expansion $\alpha$ varies in time because material properties of LIBs vary upon lithiation due to the phase transition similar to the modulus of elasticity $[43,44]$. Moreover, the equivalent coefficient of thermal expansion $\alpha_{b a t}$ is assumed to depend on temperature. This assumption, which is different to Ref. [42], is addressed for a wide range of operational temperature of battery cells. In Ref. [42], the dependency of the equivalent coefficient of thermal expansion on temperature is negligible because the temperature variation of battery cells is up to $3.5{ }^{\circ} \mathrm{C}$ in experiments. However, the coefficient of thermal expansion for many materials depends on temperature for a wide operational range of battery cells. Especially, the separators inside of the jellyrolls are made from polymer materials, which show significant temperature dependency [57-59]. Hence, the dependency of the coefficient of thermal expansion on temperature is additionally considered herein to create the high fidelity model. 


\subsection{Force estimator}

Microstructure transformation of electrodes leads to the evolution of material properties due to the phase transition $[53,54]$. Especially, the material properties for the layered structure show significant change, whereas those for the spinel and olivine structures vary minimally upon lithiation [55]. Our previous work not only showed the lithiation induced stiffening of the equivalent stiffness for a jellyroll over SOC, but also suggested the phenomenological force model [52]. The proposed force model can predict the force induced from the volume change of battery cells with free swelling measurement at constant ambient temperature for a wide range of preload condition.

In brief, the swelling over SOC was measured at unconstrained conditions, whereas the force over SOC was measured at constrained conditions with the preload and spacers. Both experiments were conducted at regulated temperature in a thermal chamber. The force was coupled with swelling with respect to SOC and governing equations were derived to identify the relationship between the force and the swelling. The model not only addressed the nonlinear elastic stiffness to capture the inherent nature of Li-ion intercalation swelling but also separated the overall SOC region into three regions to account for the phase transition. This model also addressed the initial displacement to account for the preload effect; see Ref. [52] for the details about the governing equations and coupling methods of two experiments.

The proposed force model in Ref. [52] assumed that the ambient temperature was constant and thereby the distance between two endplates was constant. However in reality, the ambient temperature of battery cells and packs varies upon weather, operation, and cooling conditions, 
suggesting that the distance between two endplates varies upon ambient temperature because of the thermal expansion/shrinkage of the frame of battery packs. Therefore, one more governing equation is derived herein to account for the variation of the distance between two endplates due to the variation of ambient temperature [35]. This modification is one of the key contributions in this study compared to Ref. [52] in the prediction of force in cells.

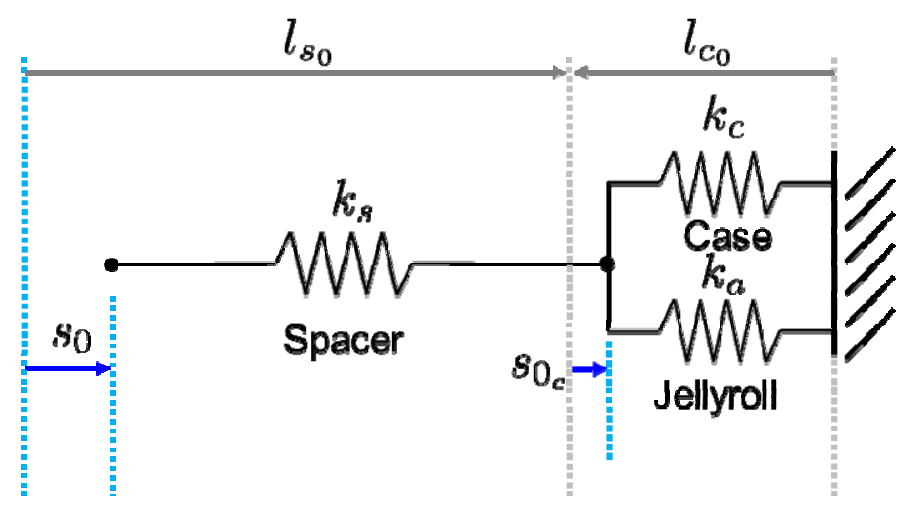

(a) Initial force equilibrium of the battery cell including the spacer with preload

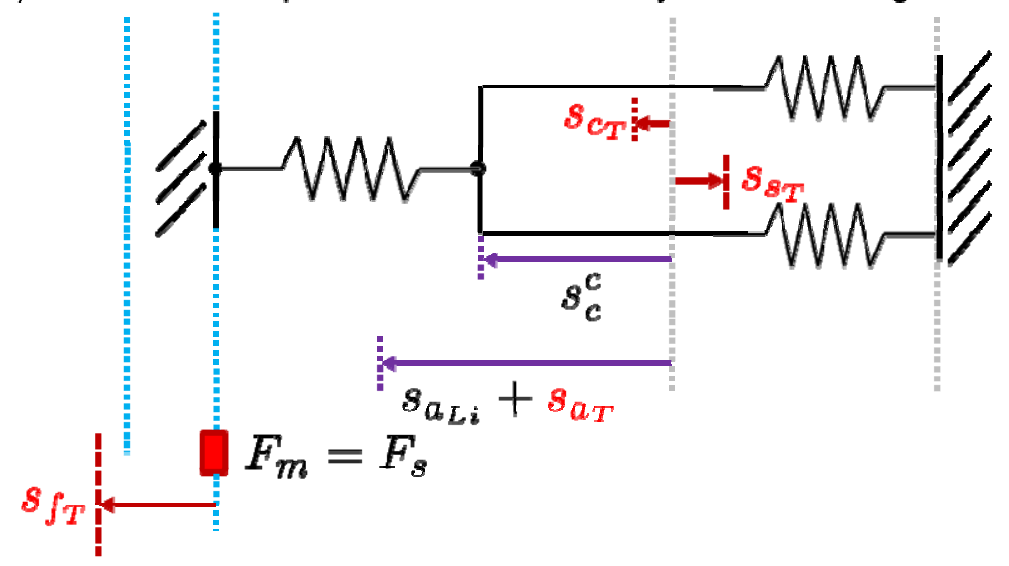

(b) Force equilibrium during operation at different ambient temperature

Figure 3. Force equilibrium for a constrained swelling of the battery cell with a plastic spacer account for the effects of the preload and temperature change; (a) initial force equilibrium at a certain ambient temperature and (b) force equilibrium at a different ambient temperature. 
Figure 3 illustrates the two force equilibriums at constrained conditions. Figure 3 (a) depicts an initial assembled condition of experiments with preload at a certain constant ambient temperature $\left(25{ }^{\circ} \mathrm{C}\right.$ in experiments of Ref. [52]). The case and the jellyroll are connected in parallel in the sense that the case impedes the expansion of the jellyroll when the jellyroll is placed inside of the case due to the stiffness of the case. The spacer is added in series with the battery cell considering the serial connection of experiments; see Figure 1 for the details of experimental setup. Then, the external load from tightening bolts causes the initial displacement for the spacer $s_{0}$ and battery cell $s_{0_{c}}$.

Figure 3 (b) illustrates the force induced from the volume change of the battery cell during lithiation/delithiation at a different ambient temperature (the first experimental sets). The ambient temperature was changed from one $\left(25^{\circ} \mathrm{C}\right)$ to the desired temperature and then force was measured during discharge. All components thermally expand when ambient temperature increases. In Figure $3(\mathrm{~b}), s_{f_{T}}, s_{s_{T}}, s_{c_{T}}$, and $s_{a_{T}}$ denote the thermal expansion of the fixture (including the bolt and Garolite endplate), the thermal expansion of the spacer, the thermal expansion of the case, and the thermal expansion of the jellyroll; $s_{a_{L i}}$ and $s_{c}^{c}$ denote the swelling of the jellyroll due to Li-ion intercalation at unconstrained conditions and the swelling of the case (and the jellyroll) at constrained conditions.

The jellyroll and the spacer are compressed, whereas the case is stretched from the original length during Li-ion intercalation in the force equilibrium. Therefore, a compressive force acts on the jellyroll and spacer, whereas a tensile force acts on the case in the force equilibrium. This force equilibrium results in 


$$
F_{s}=-F_{c}+F_{a},
$$

where $F_{s}, F_{c}$, and $F_{a}$ are the force induced from the spacer, the case, and the jellyroll respectively.

The variation of the length for the spacer is $s_{c}^{c}+s_{0}+s_{s_{T}}-s_{f_{T}}$ in the force equilibrium at a certain elevated ambient temperature. The total compressed length is $s_{c}^{c}+s_{0}+\mathrm{s}_{s_{T}}$, whereas the stretched length is $s_{f_{T}}$ from an initial length. Note that the initial length of the spacer is not $l_{s_{0}}$ but $l_{s_{0}}+\mathrm{s}_{S_{T}}$ at the certain elevated ambient temperature when unconstrained. Temperature increase changes the initial length of the spacer. Similar to the spacer, the variation of the length for the case and the jellyroll is $s_{c}^{c}-\mathrm{s}_{c_{T}}$ and $\mathrm{s}_{a_{L i}}+\mathrm{s}_{a_{T}}-s_{c}^{c}$ because the initial length of the case and the jellyroll is $l_{c_{0}}+\mathrm{s}_{c_{T}}$ and $l_{s_{0}}+\mathrm{s}_{a_{L i}}+\mathrm{s}_{a_{T}}$ considering the temperature variation at a certain charge state when unconstrained.

Applying Hook's Law for the force equilibrium (Eq. (6)), one obtains

$$
\begin{gathered}
k_{s}\left(1-\mathrm{c}_{s} \Delta \mathrm{T}\right)\left(s_{c}^{c}+s_{0}+\mathrm{s}_{s_{T}}-\mathrm{s}_{f_{T}}\right)=-k_{c}\left(s_{c}^{c}-\mathrm{s}_{c_{T}}\right)+k_{a_{1}}\left(1-\mathrm{c}_{a} \Delta \mathrm{T}\right)\left(\mathrm{s}_{a_{L i}}+\right. \\
\left.\mathrm{s}_{a_{T}}-s_{c}^{c}\right)+k_{a_{2}}\left(\mathrm{~s}_{a_{L i}}+\mathrm{s}_{a_{T}}-s_{c}^{c}\right)^{3},
\end{gathered}
$$

where $c_{s}$ and $c_{a}$ denote the dependency of the equivalent stiffness on temperature in rate (percentage) for the spacer and the jellyroll. The dependency of the equivalent stiffness on temperature for the jellyroll is assumed to be identical for overall SOC regions. The nonlinear elastic model $\left(F_{a}=k_{a_{1}} s+k_{a_{2}} s^{3}\right)$ is addressed for the jellyroll to illustrate the force dependency on swelling [52]. It is also assumed that the stiffness of the spacer and the jellyroll has a softening at elevated temperature and this softening shows a linear fashion in terms of temperature in the operation range of battery cells and packs. The polymer materials generally 
show the softening in the elasticity of modulus over temperature [56]. The spacer is made from polybutylene terephthalate (PBT), while the separator insider of the jellyroll is made from polyethylene (PE), polypropylene (PP), or combination thereof. Therefore, the stiffness of the spacer and the jellyroll can show the softening over temperature. The softening effect on the nonlinear stiffness for the jellyroll is assumed to be negligible. The nonlinear stiffness is relatively smaller than the linear stiffness and thereby the effect of softening from nonlinear terms is small considering the higher order effect, i.e. the cubic term of swelling.

The total thermal swelling of the fixture $s_{f_{T o t}}$ replaces the $s_{s_{T}}-s_{f_{T}}$ in Eq. (7). It is hard to distinguish the exact amount of thermal swelling from two components, i.e. the thermal expansion of the Garolite endplate including bolt $s_{f_{T}}$ and the spacer $s_{s_{T}}$, in the actual experiment because the equivalent length for each component is difficult to estimate because of the complex shape, especially for the spacer [35]. Moreover, the coefficient of thermal expansion for each material is unavailable. The conventional equation for thermal expansion $(\alpha L)_{f_{T o t}} \Delta T$ replaces $s_{f_{T o t}}$ in Eq. (7) because temperature variation $\Delta \mathrm{T}$ is the controllable parameter. The thermal expansion of the case can be calculated with the same formula because the coefficient of thermal expansion for aluminum, which is the material of the case, and the thickness of the case are available.

A more sophisticated approach is applied to the thermal expansion of the jellyroll. The coefficient of thermal expansion depends on SOC and temperature as aforementioned in section 3.2. Therefore, the thermal expansion of the jellyroll is expressed as $\alpha_{a}$ (SOC) $(1+\beta \Delta T) L_{a} \Delta T$. The temperature dependency of thermal swelling might be caused from separators made from a polyethylene and a polypropylene because polymer materials show significant temperature 
dependency [57-59]. The coefficient of thermal expansion for the graphite also depends on temperature, even though the value is relatively small compared with polymer materials [60]. The coefficient of thermal expansion for the jellyroll $\alpha_{a}$ (SOC) is separated into three values considering the phase transition: $\alpha_{a_{L}}$ for low SOC region (0-0.25 SOC), $\alpha_{a_{M}}$ for middle SOC region (0.5), $\alpha_{a_{H}}$ for high SOC region (0.75-1.0 SOC). This classification is followed by the trend of the equivalent coefficient of thermal expansion for the battery cell at unconstrained conditions [42]. The amplitude of thermal expansion at constrained conditions can be different than that at unconstrained conditions. However, the trend of variation over SOC can be approximately similar because this trend is caused from the inherent nature of electrochemical reaction, i.e. Li-ion intercalation and phase transition. These replacements result in

$$
\begin{gathered}
k_{s}\left(1-\mathrm{c}_{s} \Delta \mathrm{T}\right)\left(s_{c}^{c}+s_{0}+(\alpha L)_{f_{T o t}} \Delta T\right)=-k_{c}\left(s_{c}^{c}-\mathrm{s}_{c_{T}}\right)+k_{a_{1}}\left(1-\mathrm{c}_{a} \Delta \mathrm{T}\right)\left(\mathrm{s}_{a_{L i}}+\right. \\
\left.\alpha_{a}(\mathrm{SOC})(1+\beta \Delta T) L_{a} \Delta T-s_{c}^{c}\right)+k_{a_{2}}\left(\mathrm{~s}_{a_{L i}}+\alpha_{a}(\mathrm{SOC})(1+\beta \Delta T) L_{a} \Delta T-s_{c}^{c}\right)^{3} .
\end{gathered}
$$

The measured force in load cells $F_{m}$ is the same as the compression force of the spacer $F_{s}$ in the force equilibrium because load cells are installed in the one side of Garolite endplate (red filled square in Figure $3(b))$ as

$$
F_{m}=k_{s}\left(1-\mathrm{c}_{s} \Delta \mathrm{T}\right)\left(s_{c}^{c}+s_{0}+(\alpha L)_{f_{T o t}} \Delta T\right),
$$

The swelling of the case at constrained conditions can be derived in terms of the unconstrained swelling of the jellyroll, the initial displacement of the spacer, and the variation of temperature with respect to the reference temperature from Eq. (8); $s_{c}^{c}=h\left(\mathrm{~s}_{a_{L i}}, s_{0}, \Delta T\right)$ (where $h$ is a known function). The initial displacement of the spacer can be expressed in terms of that of the case (battery cell); $s_{0}=g\left(s_{0_{c}}\right)$ (where $g$ is a known function). The swelling of the jellyroll 
can be derived in terms of the measured swelling of the case at unconstrained conditions; $\mathrm{s}_{a_{L i}}=f\left(\mathrm{~s}_{c}^{f}\right)$, where $\mathrm{s}_{c}^{f}$ is the swelling of the case at an unconstrained condition and $f$ is a known function; See Ref. [52] for the details of functions $g$ and $f$. Finally, plugging three equations into (9), one obtains

$$
F_{m}=k_{s}\left(1-c_{s} \Delta \mathrm{T}\right)\left\{h\left(f\left(\mathrm{~s}_{c}^{f}\right), g\left(s_{0_{c}}\right), \Delta T\right)+g\left(s_{0_{c}}\right)+(\alpha L)_{f_{T o t}} \Delta T\right\}
$$

The measured force at constrained conditions is a function of the variation of temperature $\Delta T$, the measured swelling of the case at unconstrained conditions $\mathrm{s}_{c}^{f}$, and the initial displacement of the case due to the preload $s_{0_{c}}$. This equation suggests that the force at constrained conditions can be predictable if the variation of temperature $\Delta T$, the swelling of the case over SOC at unconstrained conditions $\mathrm{s}_{c}^{f}$, and initial displacement of the case due to preload $s_{0_{c}}$ are available. The full equation is omitted herein for the sake of brevity.

To estimate the force induced from the volume change of the battery cell for a wide range of operational ambient temperature, seven parameters should be identified; $c_{a}, c_{s},(\alpha L)_{f_{T o t}}, \alpha_{a_{L}}$, $\alpha_{a_{M}}, \alpha_{a_{H}}$, and $\beta$. Other parameters used in the model were already identified in Ref. [52]. Therefore, the parameter estimation was conducted with measured forces at the identical preload and four different ambient temperatures by using the nonlinear least square method (Figure 4 (a)). Solid lines are the nonlinear least square fitting results with identified parameters based on measurements (symbols) in Figure 4 (a). The identical initial displacement $\left(s_{0_{c}}\right)$, which was estimated from the triangle symbols of Figure 4 in Ref. [52], was used in parameter estimation. The coefficient of determination $\left(\mathrm{R}^{2}\right)$ is over 0.99 for all fitted regions, suggesting that the fitted curves are consistent with measured data in overall regions. The identified parameters are listed 
in Table 2.

Table 2. Estimates of the thermal characteristics of the model for battery cells that relate the force and swelling over SOC measured by displacement sensors and load cells.

\begin{tabular}{|c|c|c|}
\hline Parameter & Value & Unit \\
\hline $\mathrm{c}_{a}$ & 0.01 & $\% \mathrm{~K}^{-1}$ \\
\hline $\mathrm{c}_{s}$ & 3.00 & $\% \mathrm{~K}^{-1}$ \\
\hline$(\alpha L)_{f_{T o t}}$ & $1.63 \times 10^{-6}$ & $\mathrm{mK}^{-1}$ \\
\hline$\alpha_{L}$ & 1.42 & $1 \mathrm{e}-4 \mathrm{~K}^{-1}$ \\
\hline$\alpha_{M}$ & 1.20 & $1 \mathrm{e}-4 \mathrm{~K}^{-1}$ \\
\hline$\alpha_{H}$ & 1.32 & $1 \mathrm{e}-4 \mathrm{~K}^{-1}$ \\
\hline$\beta$ & 2.5 & $\% \mathrm{~K}^{-1}$ \\
\hline
\end{tabular}

The estimated coefficient of thermal expansion for a jellyroll $\alpha_{*}$ for different SOCs is similar in order of magnitude. However, the maximum difference is around $15 \%$, suggesting that the difference from the phase transition is not negligible. Moreover, the temperature dependency of the coefficient of thermal expansion $\beta$ is not small and is essential to predict the dynamic response of battery cells for a wide range of operational temperature. The equivalent coefficient of thermal expansion for the battery cell $\alpha_{b a t}(T, t)$, which is used to estimate the thermal swelling of the battery cell in section 3.2, is calculated with the coefficient of thermal expansion and the thickness of the jellyroll and the case (Figure 4 (c)) as

$$
\alpha_{b a t}(T, t) L_{b a t} \Delta T=\alpha_{c} L_{c} \Delta T+\alpha_{a}(T, t) L_{a} \Delta T
$$

where $\alpha_{c}, L_{c}$ denote the coefficient of thermal expansion and the thickness of the case made from aluminum. The coefficient of thermal expansion for a battery cell is also linearly proportional to temperature, but the magnitude of the coefficient of thermal expansion for a 
battery cell is $9 \%$ smaller than that for a jellyroll because of the small coefficient of thermal expansion of the aluminum case.

The softening of the stiffness for the spacer is significant, while that for the jellyroll is negligible. One plausible explanation is that polymer material of the separators in battery cells is small portion below $30 \%$, whereas whole spacer is made from PBT. Therefore, the softening originated from polymer materials is more severe in the spacer. The other explanation is that PBT shows more significant temperature dependency than a polyethylene and a polypropylene. However, the exact origin for these results is hard to explain before characterizing the thermal characteristics of individual materials. Note that the material properties of polymer materials significantly depend on many things such as the atomic structure, the density of materials, and the manufacturing process even for the same material. Therefore, polymers show a wide range of variation, which is why research has been intensively carried out to elucidate the material properties of individual polymer materials [56].

Note also that measuring all thermal characteristics of individual materials is very time and cost intensive. The purpose of this study is to create the phenomenological multi-physics model on the cell-level for control-oriented purpose, not to identify the thermal characteristics of individual components. 

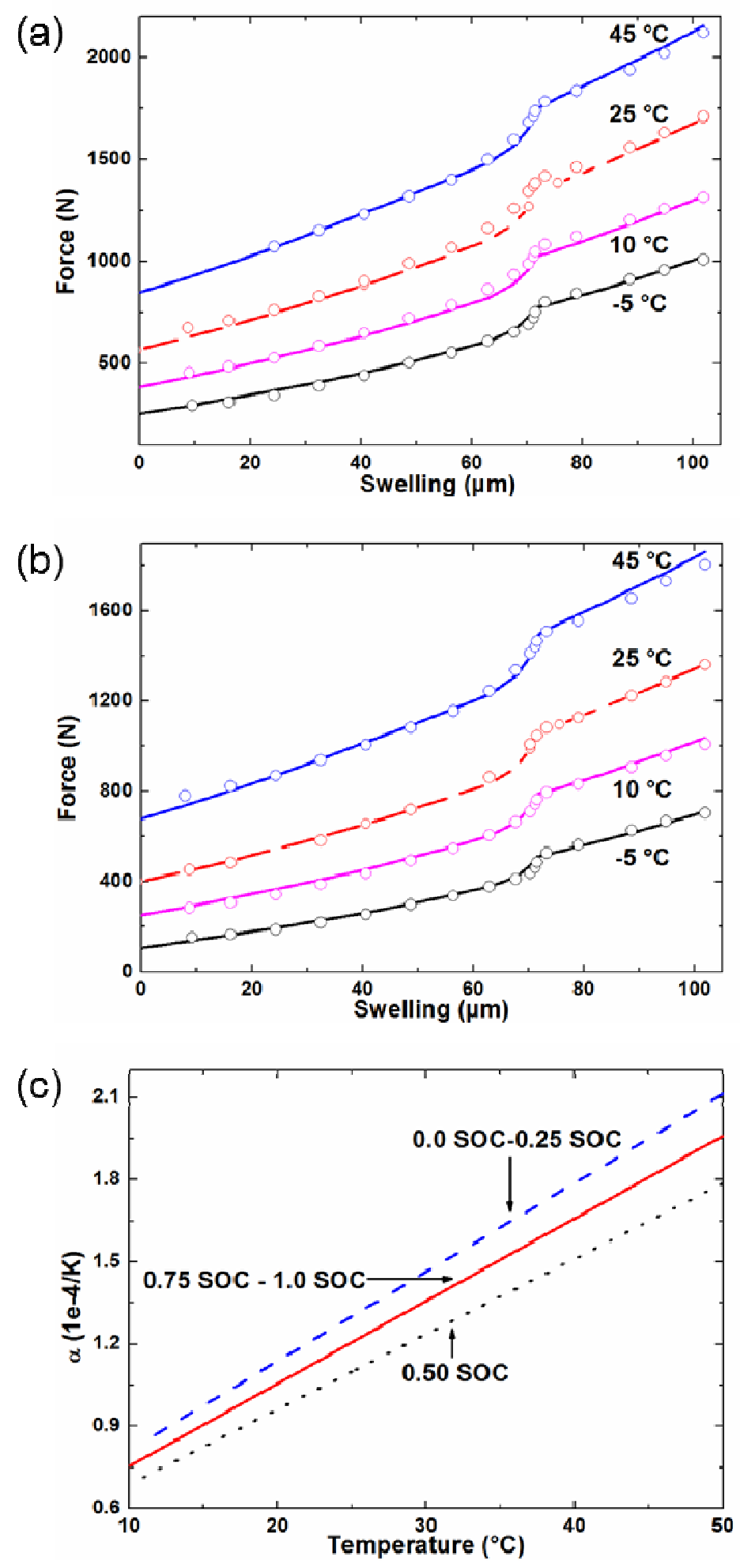

Figure 4. Force versus swelling at several ambient temperatures with the preload of (a) $670 \mathrm{~N}$ and (b) $450 \mathrm{~N}$ (at $0.05 \mathrm{SOC}$ and $25^{\circ} \mathrm{C}$ temperature); the solid lines are forces predicted from the 
model, whereas the circle symbols are forces measured in (a) and (b) of the figure. (c) The coefficient of thermal expansion of the battery cell over temperature at different SOCs.

\section{Validation}

Intensive experimental validation was carried out to verify the force prediction. The ETM has already been validated at a variety of operational conditions in Ref. [39]. Section 4.1 compares the predicted quasi-static force induced from the Li-ion intercalation to experiments at a variety of ambient temperature. Section 4.2 presents the model prediction of force due to the thermal expansion of battery cells and compares to pulse excitation experiments at three different internal charge states. Finally, the proposed model was validated through experiments where the power split from a fusion HEV traversing the US06 drive cycle was applied at different SOCs, preloads, and ambient temperatures in section 4.3 .

\subsection{Force induced from Li-ion intercalation in a steady state}

The model predictions were compared to the force measured with a different preload than preload used for the parameter identification in section 3 at several ambient temperatures for the validation of parameters identified (Figure $4(\mathrm{~b})$ ). The identical initial displacement of the case $s_{0_{c}}$, which was estimated from the circle symbols of Figure 4 in Ref. [52], was applied for the force model in this verification with the same parameters listed in Table 1 because the identical 
preload of the circle symbols of Figure 4 in Ref. [52] was used in these experiments. The initial displacement of the case $s_{0_{c}}$ is only a parameter changed from the data used in the parameter identification to verify the parameters. Even the initial displacement estimated from previous work was used. The solid lines are model predictions, whereas the symbols are experiments in Figure 4 (b). The surface temperature of battery cells is not shown herein because the temperature of battery cells is identical to ambient temperature in a steady state. The model prediction is in excellent agreement with measured data, suggesting that estimated parameters are accurate and reliable, and thereby the proposed model can predict the electrochemicalinduced force for a wide range of preload and ambient temperature at the steady state. Moreover, this result justifies the hypothesis that the Li-ion induced swelling of battery cells over SOC is constant regardless of temperature in the range of experiments.

\subsection{Force induced from temperature elevation}

The surface temperature and force from temperature elevation, which are estimated from the proposed multi-physics model, are compared to second experimental sets; pulse excitation experiments at three different internal charge states: $0.22 \mathrm{SOC}, 0.48 \mathrm{SOC}$, and $0.74 \mathrm{SOC}$ (Figure $5)$. 
(a)

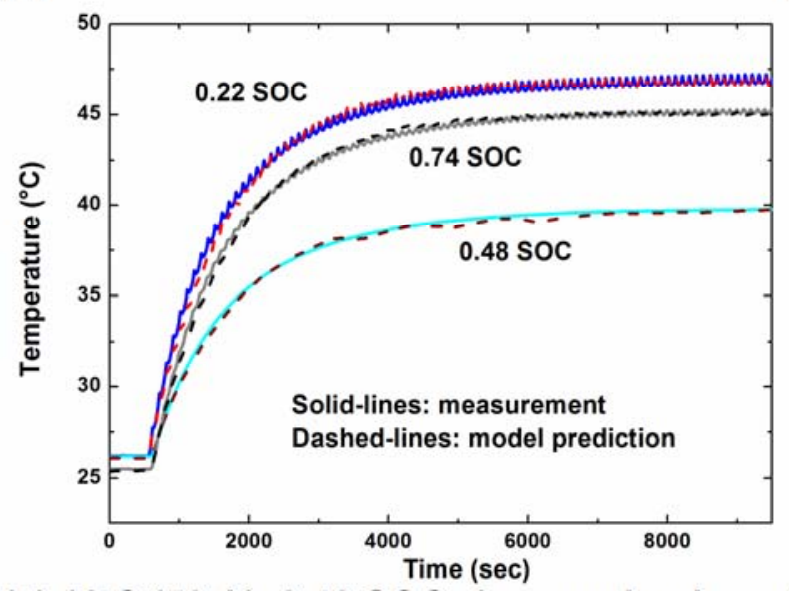

(c) $10 \mathrm{C}(50 \mathrm{~A}), 0.48 \mathrm{SOC}, 1$ second pulse

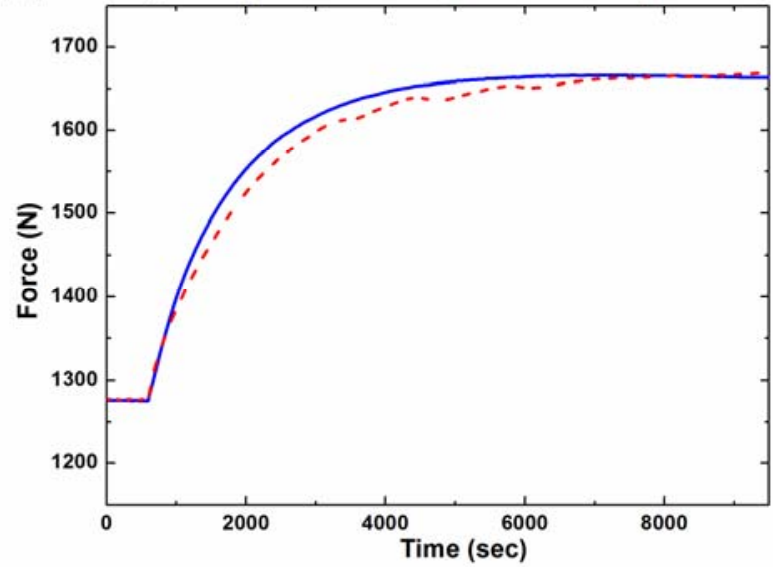

(b) $10 \mathrm{C}(50 \mathrm{~A}), 0.22 \mathrm{SOC}, 100$-second pulse

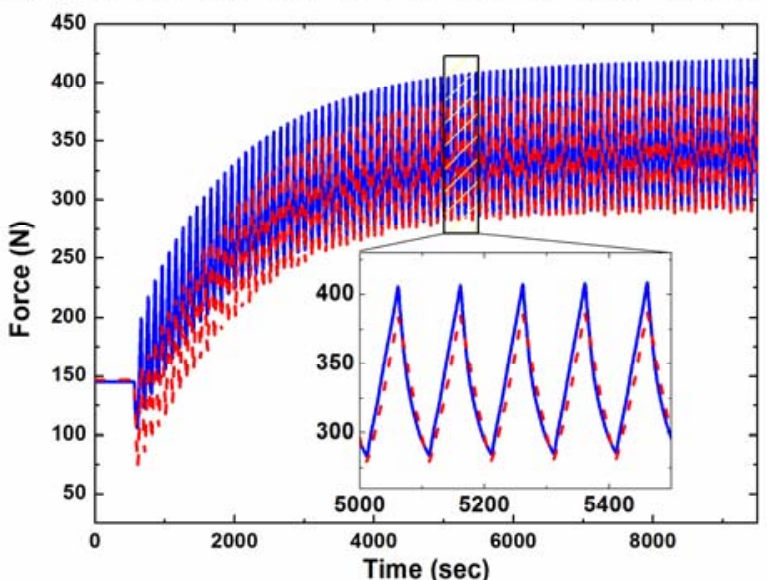

(d) $10 \mathrm{C}(50 \mathrm{~A}), 0.74 \mathrm{SOC}, 100$-second pulse

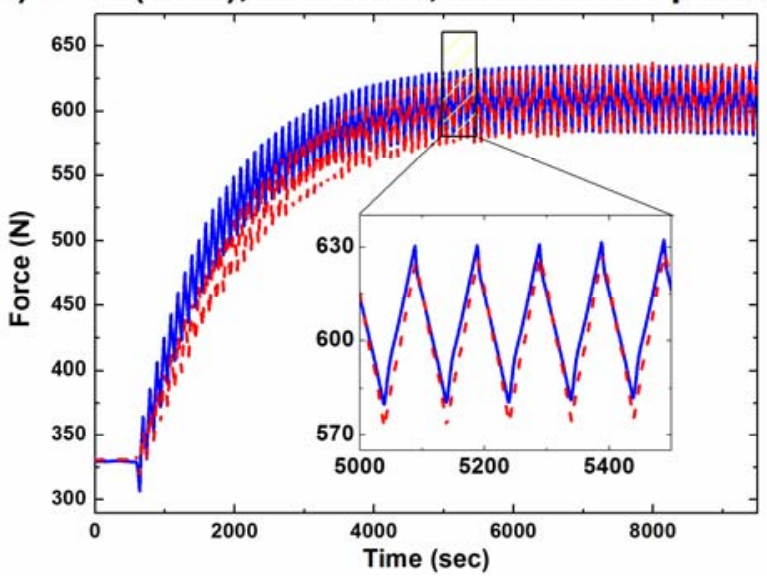

Figure 5. (a) Surface temperature and force of the battery cell during pulse excitation at (b) 0.22 SOC, (c) 0.48 SOC, and (d) 0.74 SOC; the solid lines are the surface temperature measured, whereas the dashed lines are the surface temperature predicted in figure (a); the solid lines are the force measured, whereas the dashed lines are the force predicted in figure (b)-(d).

The surface temperature of the battery cell is presented in Figure 5 (a). Solid lines are measured ones, whereas dashed lines are predicted by the proposed model in Figure 5 (a). The heat convection coefficient was slightly tuned around $10 \mathrm{Wm}^{-1} \mathrm{~K}^{-1}$ for each case when estimating temperature of cells in the ETM model because the heat convection coefficient depends on the 
location of experimental setup even in the same thermal chamber; the flow rate is different in the same chamber and depends on the location of experimental setup. The long period of pulse excitation results in the slight triangular fluctuation with the first-order response in the surface temperature at $0.22 \mathrm{SOC}$ and $0.74 \mathrm{SOC}$, whereas the short period of pulse excitation causes the first-order response. These phenomena can be explained by the fact that entropy change plays a role in the long period of pulse excitation, whereas this effect is negligible in the short period of pulse excitation. Entropy heat is endothermic, i.e. heat sink, during charge and exothermic, i.e. heat source, during discharge. Moreover, the magnitude of triangular fluctuation at $0.22 \mathrm{SOC}$ is different than that at $0.74 \mathrm{SOC}$ because the value of $\frac{d U}{d T}$ is the slope of the OCV as a function of temperature at that SOC. It can be inferred that the short period of pulse excitation is better to obtain the pure thermal response. The root-mean-square errors (RMSE) in the surface temperature are $0.25{ }^{\circ} \mathrm{C}, 0.20{ }^{\circ} \mathrm{C}$, and $0.21{ }^{\circ} \mathrm{C}$ at 0.22 SOC, 0.48 SOC, and 0.74 SOC respectively. This difference is similar in order of magnitude with the results in Ref. [39].

The estimated forces induced from thermal expansion are compared to experiments (Figure 5 (b)-(d)). The solid lines are the force measured, whereas the dashed lines are the force predicted in Figure 5 (b)-(d). Different SOCs and preloads were induced to the experimental setup to validate the model prediction for a wide range of preloads and SOCs. The initial displacements were estimated with initial measured forces and SOCs for each case by using Eq. (10). The measured force at 0.48 SOC (Figure 5 (c)) clearly shows the first-order response similar to surface temperature, suggesting that this reaction force is originated from the thermodynamics of Li-ion cells. This result confirms again that the short period of pulse excitation minimally changes the SOC and thereby results in pure thermal expansion and its induced force. On the 
other hand, the long period of pulse excitation accompanies the variation in SOC as shown in the measured force at $0.22 \mathrm{SOC}$ (Figure 5 (b)) and 0.74 SOC (Figure 5 (d)). The wide fluctuation from Li-ion intercalation is shown together with the first-order response, which is driven by the temperature elevation. Moreover, this result not only justifies the hypothesis that the coefficient of thermal expansion for the jellyroll depends on SOC, but also validates that the estimated coefficient of thermal expansion is accurate and reliable. The model predictions are in good agreement with experiments in all SOC regions. The RMSE in the predicted force are $16.2 \mathrm{~N}$, $11.7 \mathrm{~N}$, and $13.9 \mathrm{~N}$ at $0.22 \mathrm{SOC}, 0.48 \mathrm{SOC}$, and $0.74 \mathrm{SOC}$ respectively.

The relatively wide fluctuation is shown at 0.22 SOC pulse excitation experiment compared to that at 0.74 SOC pulse excitation experiment even though the preload induced is smaller; the magnitude in peak-to-peak due to $\mathrm{Li}$-ion intercalation is over $100 \mathrm{~N}$ at $0.22 \mathrm{SOC}$, whereas that is around $50 \mathrm{~N}$ at $0.74 \mathrm{SOC}$. This observation can be explained by the amount of Li-ion intercalation swelling. The amount of swelling from Li-ion intercalation is $22 \mu \mathrm{m}$ when SOC fluctuates in the range of $0.09 \mathrm{SOC}$ to $0.22 \mathrm{SOC}$, while that from Li-ion intercalation is $4 \mu \mathrm{m}$ when SOC fluctuates in the range of $0.60 \mathrm{SOC}$ to $0.74 \mathrm{SOC}$, suggesting that the relatively large variation of $\mathrm{Li}$-ion intercalation swelling in low SOC causes the wide fluctuation of reaction forces. The swelling due to Li-ion intercalation gently increases in the range of $0.45 \mathrm{SOC}$ to 0.75 SOC (inset figure on the left-bottom side of Figure 2).

\subsection{US06 duty cycle}


Five operational conditions were tested with the US06 duty cycle for the validation of the dynamic response (Table 1). In the first case, the battery cell was operated with a certain preload and the initial SOC of 0.50 at $25{ }^{\circ} \mathrm{C}$ ambient temperature. In the second case, the same configuration, the same preload and initial SOC, was used except ambient temperature. This case ran at $11{ }^{\circ} \mathrm{C}$ ambient temperature. In the third case, the initial SOC and preload were reduced, whereas ambient temperature was kept to $11{ }^{\circ} \mathrm{C}$ ambient temperature. In the fourth and fifth experiments, preload was more reduced; the preload induced was almost half of the first and second cases. Two different initial SOCs were tested at $11{ }^{\circ} \mathrm{C}$ ambient temperature.

The temperature dependency of parameters was validated through the first and second experiments because these experiments were carried out with the identical preload and SOC. The dynamic force response for a wide range of force was verified through the second to fourth experiments. Finally, the dynamic force response for a wide range of SOC was validated through the third to fifth experiments. 


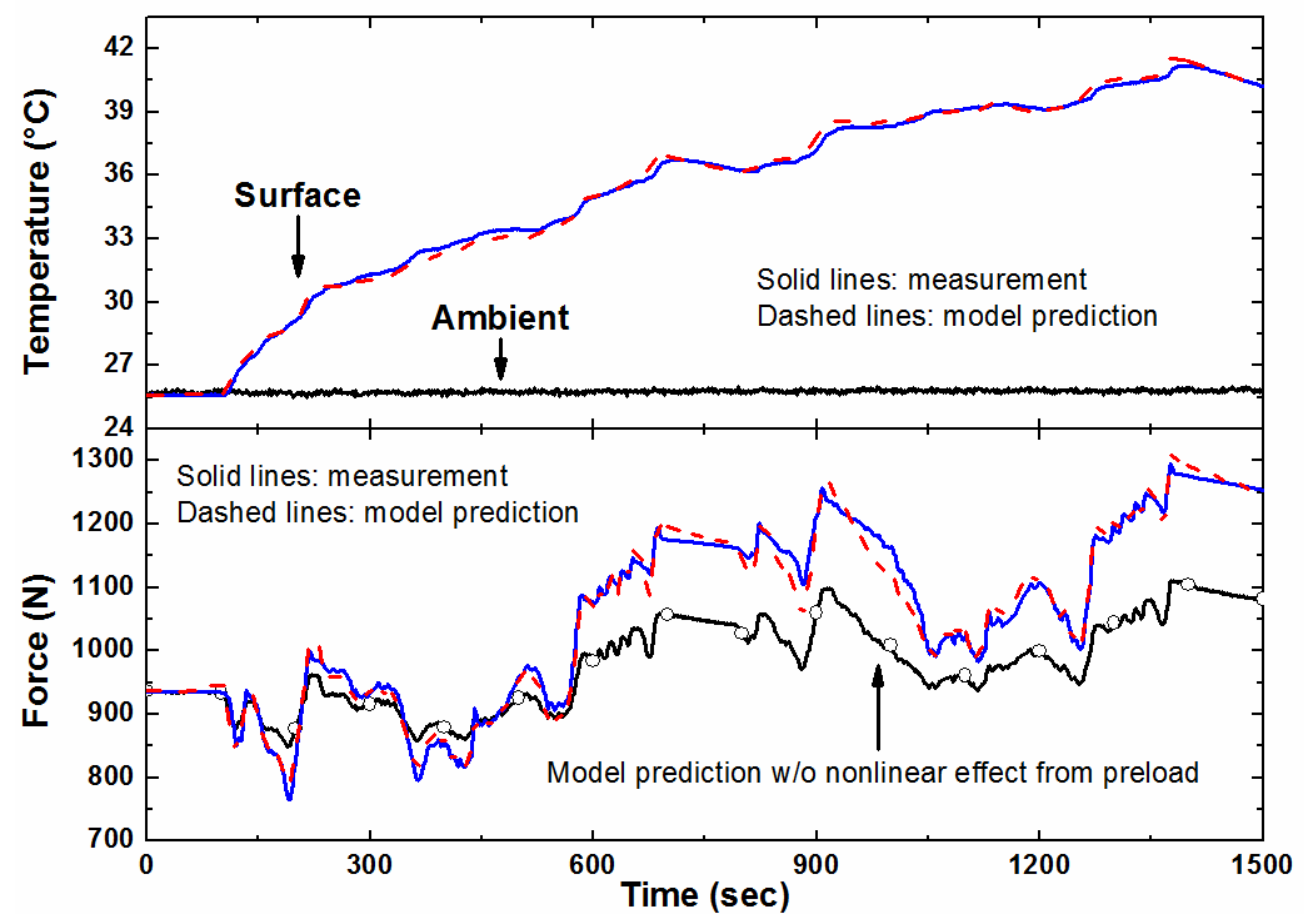

Figure 6. (Top) Temperature and (bottom) force of the battery cell with initial 0.50 SOC during the US06 duty cycle at $25{ }^{\circ} \mathrm{C}$ ambient temperature; the solid-line is the surface temperature measured, whereas the dashed line is the surface temperature predicted in the top of the figure; the solid line, the dashed line, and the symbols with line denote the force measured, the force predicted including the nonlinear effect from preload, and the force predicted without nonlinear effect from preload in the bottom of the figure.

Figure 6 shows the comparison between measurement and model prediction for the first experiment of Table 1. The solid-line is the surface temperature measured, whereas the dashed line is the surface temperature predicted in the top of Figure 6. The solid line, the dashed line, and the symbols with the line denote the force measured, the force predicted including the nonlinear effect from preload, and the force predicted without nonlinear effect from preload in the bottom of Figure 6. The surface temperature estimated is in excellent agreement with the 
surface temperature measured. The RMSE in the prediction of surface temperature is $0.19{ }^{\circ} \mathrm{C}$. Two conditions were simulated in the estimation of force to elucidate the nonlinear effect from preload. The first set of results assumed that the initial displacement was zero and calculated force trends for overall period. Then, a certain amount of force $(940 \mathrm{~N})$ was added to the calculated force trends to match the initial force (the symbols with line in the bottom of Figure 6). The second set of results estimated force by using the Eq. (10) to accurately predict the force including nonlinear effect, which is originated from preload (the dashed line in the bottom of Figure 6). This comparison clearly demonstrates the importance of the nonlinear effect from preload. The RMSE of the force estimation for the first set is $97.6 \mathrm{~N}$, whereas the RMSE of the force estimation for the second set is $16.1 \mathrm{~N}$. These errors correspond to $7.5 \%$ and $1.2 \%$ with respect to the maximum force measured in this experiment. The error from the model prediction without the nonlinear effect from preload is over six times larger than the error from the accurate model prediction, suggesting that initial displacement from the preload plays a critical role in the estimation of the force and thereby the nonlinear effect from preload should be taken into account in the model.

Figure 7 shows the second and third experiments of Table 1 together with the estimation of the proposed model. These experiments were tested at $11^{\circ} \mathrm{C}$ ambient temperature as aforementioned. However, the second one used the identical SOC and preload with those of the first experiment, whereas the third one used different SOC and preload. The solid lines show the surface temperature measured, while the dashed lines represent the surface temperature predicted in the top of Figure 7. The prediction of surface temperature is in excellent agreement similar to other cases. The RMSE in the prediction of surface temperature is $0.20{ }^{\circ} \mathrm{C}$ for the second case and $0.19^{\circ} \mathrm{C}$ for the third case. The multi-physics model also accurately predicts the force for the 
overall period of experiments (the bottom of Figure 7). The solid lines show the force measured, while the dashed lines represent the force predicted in the bottom of Figure 7. The RMSE in the prediction of force is $19.4 \mathrm{~N}$ for the second case and $12.2 \mathrm{~N}$ for the third case.

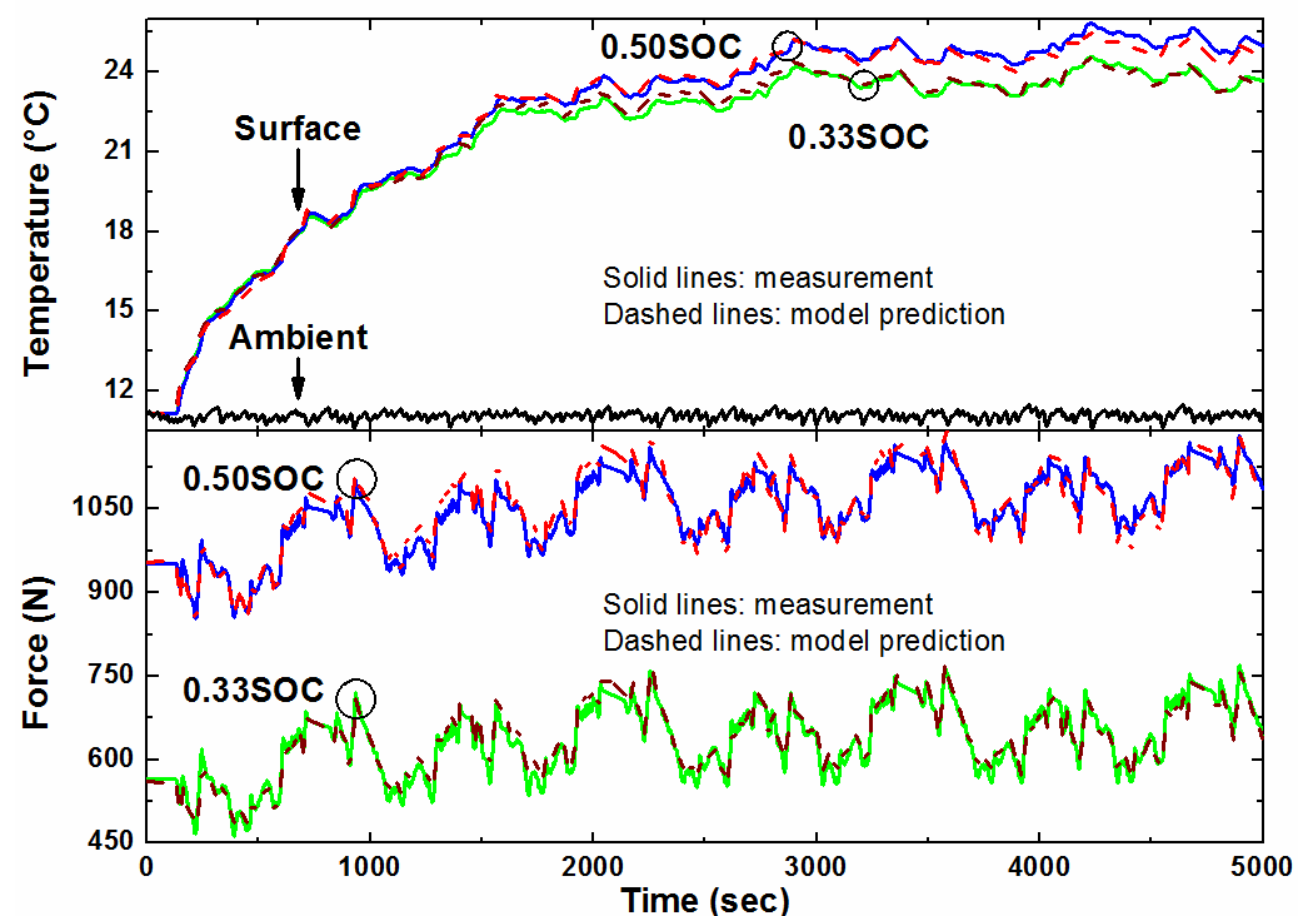

Figure 7. (Top) Temperature and (bottom) force of the battery cell during the US06 duty cycles with different preload and different $\mathrm{SOC}$ at $11{ }^{\circ} \mathrm{C}$ ambient temperature; the solid lines are the surface temperature measured, whereas the dashed lines are the surface temperature predicted in the top of the figure; the solid lines are the force measured, whereas the dashed lines are the force predicted in the bottom of the figure.

Figure 8 illustrates the fourth and fifth experiments of Table 1 with the prediction of force. These experiments were tested with the same preload at $11{ }^{\circ} \mathrm{C}$ ambient temperature. However, the fourth one was operated with the initial SOC of 0.50 , whereas the fifth one was operated with the initial SOC of 0.66. The temperature measured is shown as the solid lines, while the temperature 
predicted is represented as the dashed lines in the top of Figure 8 . The surface temperature predicted corresponds well with the surface temperature predicted. The RMSE in the prediction of surface temperature is $0.18{ }^{\circ} \mathrm{C}$ for the fourth case and $0.22{ }^{\circ} \mathrm{C}$ for the fifth case. The dynamic response of force is shown in the bottom of Figure 8. The solid lines show measurement, whereas the dashed lines represent model prediction. In two simulations, the same initial displacement was used and other parameters used were identical. The force predicted is also in good agreement with the force measured. The RMSE in the prediction of force is $15.8 \mathrm{~N}$ for the fourth case and $7.6 \mathrm{~N}$ for the fifth case.

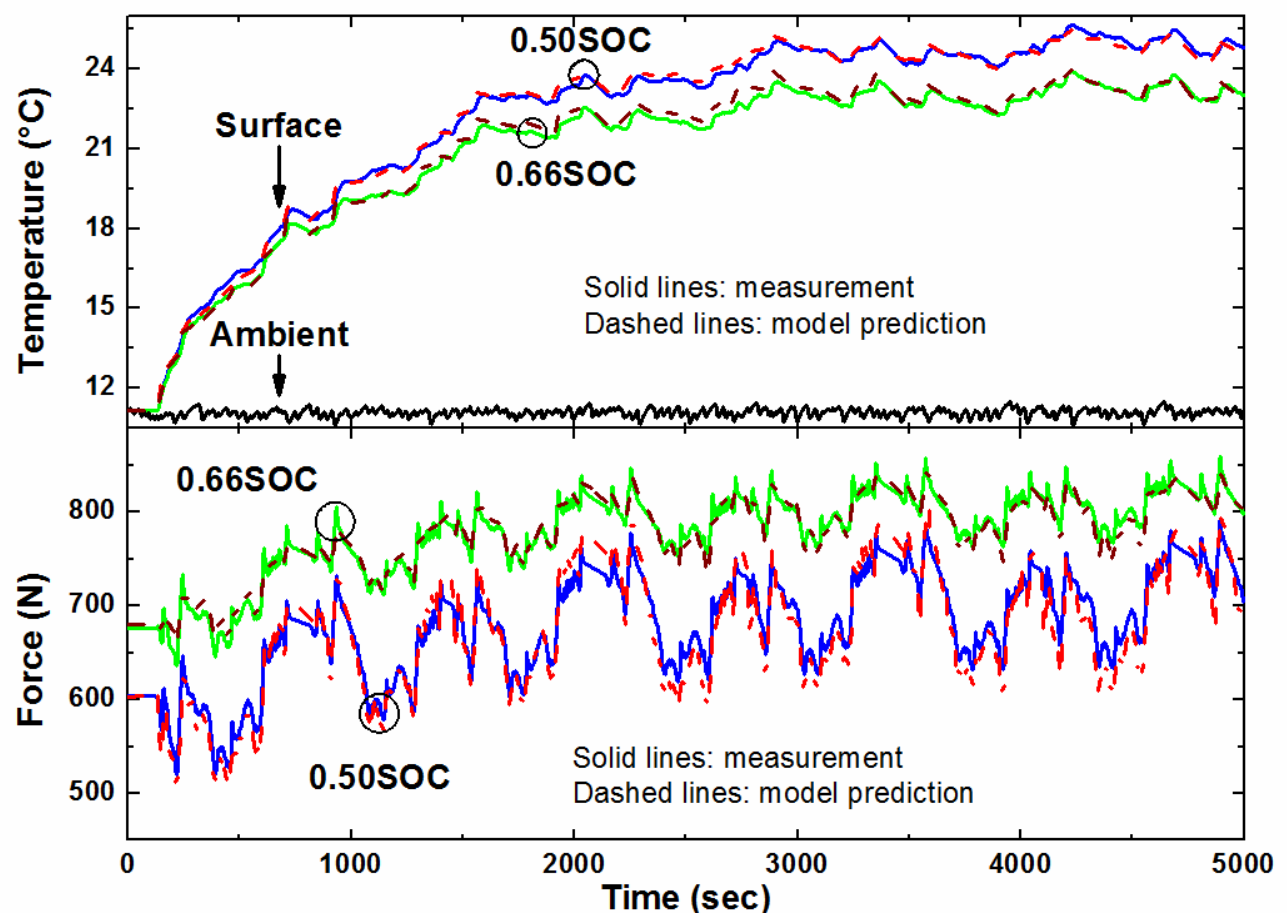

Figure 8. (Top) Temperature and (bottom) force of the battery cell during the US06 duty cycles with the same preload and different SOC at $11{ }^{\circ} \mathrm{C}$ ambient temperature; the solid lines are the surface temperature measured, whereas the dashed lines are the surface temperature predicted in the top of the figure; the solid lines are the force measured, whereas the dashed lines are the 
force predicted in the bottom of the figure.

\subsection{Summary and discussion}

The first experiment demonstrates the importance of nonlinear effect from preload. Therefore, the model accounted for nonlinear effect should be used in BMSs for effective power and thermal management. The first and second experiments show that the proposed model can operate regardless of ambient temperature. The experimental conditions for the first and the second experiments are identical except ambient temperature and thereby use the same parameters including the identical initial displacement to estimate the dynamic response of force in the model. These results suggest that the force model proposed is capable of the distance variation of two endplates with respect to temperature change as well as the dependency of the stiffness and the coefficient of thermal expansion on temperature. The results of the fourth and the fifth experiments show that the model with appropriate initial displacement accurately predicts the dynamic response of force with the same parameters. Moreover, the capability of the force model for a wide range of SOC and preload were verified through the second to fifth experiments.

A total of 16 different NMC cells were used in this study. For the development of the ETM (mentioned in Section 3.1), a single fixture composed of 3 cells was used [38]. For the development of swelling models, a fixture was devised to measure the swelling on a single cell $[42,52]$. For the development of force model and the validation of the multi-physics model, 4 different fixtures (Figure 1) were used with different initial SOC and preloading; each fixture consists of 3 cells. As mentioned in Section 4.3, the maximum RMSE between the model 
prediction and measurement is $19.4 \mathrm{~N}$ for the force and $0.22{ }^{\circ} \mathrm{C}$ for the surface temperature although each US06 cycle ran at different fixture with different cells. The variation in temperature is negligible considering the accuracy of RTDs $\left(0.5^{\circ} \mathrm{C}\right)$. The variation of force is less than $2 \%$ of the preload of battery packs considering that normal preload for battery packs is higher than $1000 \mathrm{~N}$. Hence, it can be inferred that the proposed model can accurately predict the complex behaviors of the battery cell used in the Ford Fusion HEV, even though cell-to-cell variation exists in cells. Note that this study uses only one type of NMC cells for building the model.

The purpose of this study is to introduce a general work-frame for developing fully coupled electro-thermal-mechanical models of Li-ion batteries. The proposed modeling approach can be applied to other types of Li-ion cells that experience measurable strain/stress due to Li-ion intercalation and temperature variation with the change of parameters used because all materials have stiffness to construct their micro-structure. The proposed approach consists of creating, calibrating, and integrating: (a) an ETM model, with (b) a swelling model due to Li-ion intercalation model, with (c) a thermal swelling model, and with (d) a force model. This is a general approach that can be applied to other battery systems, beyond the type and maker of the Li-ion cells that we selected for the demonstrative implementation. Future work includes implementing this method for battery systems with other types of Li-ion cells.

Our modeling approach has three important aspects for the industrialization of $\mathrm{Li}$-ion batteries: 1) an accurate SOC estimation, 2) a potential increase in battery utilization, and 3) a possible reduction in the number of batteries and sensors in a battery pack. When it comes to hybrid electric vehicles, the window for battery SOC usage is generally limited between $40 \%$ and $60 \%$ to ensure power capability. This conservative SOC window is used to prevent unfavorable 
discharging or overcharging. When accurate information about Li-ion batteries is available, the SOC window can be widened and thereby battery utilization can be increased. As discussed in Ref. [61], the mean absolute error in the estimation of SOC using force measurements could be at least half of that when using voltage measurements alone. Consequently, the number of batteries used in a battery pack could be reduced without sacrificing power capability as discussed in Ref. [62]. Lastly, the effectiveness of using force in estimating SOC-imbalance between batteries connected in series has been addressed in Ref. [63]. Ref. [63] has demonstrated that SOC-imbalance between two batteries can be detected with a single force sensor. This could potentially lead to a decrease in the total number of sensors and improve the accuracy of SOC estimation. Ultimately, the proposed modeling approach enables advanced control techniques and real-time condition monitoring for BMSs, suggesting that the reliability and safety of battery cells and packs can be improved.

\section{Conclusions}

Predicting sensitive metrics such as temperature and electrochemical- and thermal-induced strain and stress can provide useful information to estimate SOC in battery cells and packs. For these purposes, this study proposes the fully coupled phenomenological multi-physics model for innovative power and thermal management strategies in the next generation BMSs. The parameters describing thermal and mechanical mechanics on the cell-level are estimated through experimental data because measuring all thermal and mechanical characteristics of individual materials is time and cost intensive. Mechanical constrains and geometric complexities also 
affect the mechanical responses including swelling and swelling-induced force, suggesting that addressing lumped parametric models is an efficient approach for control-oriented purposes. The intensive experimental validation at a variety of operational conditions demonstrates that the proposed model accurately predicts the surface temperature and the reaction force from the volume change of the battery cell for a wide range of preload and ambient temperature. Future work includes implementing this method on other types of Li-ion cells and estimating SOC based on fusing information from voltage, temperature, or force measurements.

\section{Acknowledgements}

The information, data, or work presented herein was funded in part by the Advanced Research Projects Agency-Energy (ARPA-E), U.S. Department of Energy, under Award Number DEAR0000269.

\section{Disclaimer}

The information, data, or work presented herein was funded in part by an agency of the United States Government. Neither the United States Government nor any agency thereof, nor any of their employees, makes any warranty, express or implied, or assumes any legal liability or responsibility for the accuracy, completeness, or usefulness of any information, apparatus, product, or process disclosed, or represents that its use would not infringe privately owned rights. Reference herein to any specific commercial product, process, or service by trade name, trademark, manufacturer, or otherwise does not necessarily constitute or imply its endorsement, recommendation, or favoring by the United States Government or any agency thereof. The views 
and opinions of authors expressed herein do not necessarily state or reflect those of the United States Government or any agency thereof.

\section{References}

[1] M. Bryner, Chemical Engineering Progress. 109 (10) (2013) 36-39.

[2] A. N. Jansen, Chemical Engineering Progress. 109 (10) (2013) 57-64.

[3] B. Scrosati, and J. Garche, Journal of Power Sources. 195 (2010) 2419-2430.

[4] V. Ramadesigan, P. W. C. Northrop, S. De, S. Santhanagopalan, R. D. Braatz, and V. R. Subramanian. Journal of the Electrochemical Society. 159 (3) (2012) R31-R45.

[5] K.-Y. Oh, J. B. Siegel, L. Secondo, S. U. Kim, N. A. Samad, J. Qin, D. Anderson, K. Garikipati, A. Knobloch, B. I. Epureanu, C. W. Monroe, and A. G. Stefanopoulou, Journal of Power Sources. 267 (2014) 197-202.

[6] M. Doyle, T. Fuller, and J. Newman, Journal of the Electrochemical Society. 140 (6) (1993) 1526-1533.

[7] K. Murashko, J. Pyrhonen, and L. Laurila, IEEE Transactions on Energy Conversion. 28 (2) (2013) 335-343.

[8] M. Einhorn, F. Conte, C. Kral, and J. Fleig, IEEE Transactions on Power Electronics. 28 (3) (2013) 1429-1437.

[9] Y. Hu, S. Yurkovich, Y. Guezennec, and B. Yurkovich, Journal of Power Souces. 196 (1) (2011) 449-457.

[10] S.-C. Chen, Y.-Y. Wang, and C.-C. Wan, Journal of the Electrochemical Society. 153 (4) (2006) A637-A648. 
[11] S. Peck, T. Olszanski, S. Zanardelli, and M. Pierce, SAE Int. J. Passeng. Cars - Electron. Electr. Syst. 5 (1) (2012) 154-163.

[12] V. Srinivasan, and C. Y. Wang, Journal of the Electrochemical Society. 150 (1) (2003) A98A106

[13] Y. Chen, and J. W. Evans. Journal of the Electrochemical Society. 143 (9) (1996) 27082712.

[14] Y. Kim, S. Mohan, J. B. Siegel, A. G. Stefanopoulou, and Y. Ding, IEEE Transactions on control systems technology. 22 (6) (2014) 2277-2286.

[15] X. Lin, H. E. Perez, J. B. Siegel, A. G. Stefanopoulou, Y. Li, R. D. Anderson, Y. Ding, and M. P. Castanier, IEEE Transactions on Control System Technology. 21 (5) 2013 1745-1755.

[16] X. Lin, H. E. Perez, S. Mohan, J. B. Siegel, A. G. Stefanopoulou, and Y. Ding, and M. P. Castanier, Journal of Power Sources. 257 (2014) 1-11.

[17] X. Hu, S. Lin, S. Stanton, and W. Lian, IEEE Transactions on Industry Applications. 47 (4) (2011) 1692-1699.

[18] M.-S. Wu, K. H. Liu, Y.-Y. Wang, and C.-C. Wan, Journal of Power Sources. 109 (2002) $160-166$.

[19] H. Park, Journal of Power Sources. 239 (2013) 30-36.

[20] F. He, H. Wang, and L. Ma, International Journal of Heat and Mass Transfer. 91 (2015) 630-639.

[21] G.-H. Kim, K. Smith, K.-J. Lee, S. Santhanagopalan, and A. Pesaran, Journal of Power Souces. 158 (8) (2011) A955-A969.

[22] Y. Xie, J. Li, and C. Yuan, Journal of Power Sources. 248 (2014) 172-179.

[23] S. Jung, D. Kang, Journal of Power Sources. 248 (2014) 498-509. 
[24] S. U. Kim, P. Albertus, D. Cook, C. W. Monroe, and J. Christensen, Journal of Power Sources. 268 (2014) 625-633.

[25] J.B. Siegel, A.G. Stefanopoulou, P. Hagans, Y. Ding, and D. Gorsich, Journal of the Electrochemical Society. 160 (8) (2013) A1031-1038A.

[26] X. Wang, Y. Sone, G. Segami, H. Naito, C. Yamada, and K. Kibe, Journal of the Electrochemical Society. 154 (1) (2007) A14-A21.

[27] N. Zhang, and H. Tang, Journal of Power Sources. 218 (2012) 52-55.

[28] D. Liu, Y. Wang, Y. Xie, L. He, J. Chen, K. Wu, R. Xu, and Y. Gao, Journal of Power Sources. 232 (2013) 29-33.

[29] F. Kaasik, T. Tamm, M. M. Hantel, E. Perre, A. Aabloo, E. Lust, M. Z. Bazant, and V. Presser, Electrochemistry Communications. 34 (2013) 196-199.

[30] J. Christensen, Journal of the Electrochemical Society. 157 (2010) A366-A380.

[31] J. Christensen, and J. Newman, Journal of Solid State Electrochemistry. 10 (5) (2006) 293319.

[32] J. Christensen, and J. Newman, Journal of the Electrochemical Society. 153 (2006) A1019A1030.

[33] J. Cannarella, and C. B. Arnold, Journal of Power Sources. 245 (2014) 745-751.

[34] J. Cannarella, and C. B. Arnold, Journal of Power Sources. 269 (2014) 7-14.

[35] S. Mohan, Y. Kim, J. B. Siegel, N. A. Samad, and A. G. Stefanopoulou, Journal of the Electrochemical Society. 161 (14) (2014) A2222-A2231.

[36] N. Zhang, and H. Tang, Journal of Power Sources. 218 (2012) 52-55.

[37] B. Sood, M. Osterman, and M. Pecht, 2013 IEEE Symposium on Product Compliance Engineering. Austin, USA (2013). 
[38] N. A. Samad, J. B. Siegel, and A. G. Stefanopoulou, ASME 2014 Dynamic Systems and Control Conference, San Aonotio, USA (2014).

[39] N. A. Samad, B. Wang, J. B. Siegel, and A. G. Stefanopoulou, Journal of Dynamic Systems, Measurement, and Controls, under revision.

[40] H. He, R. Xiong, and J. Fan, Energies. 4 (2011) 582-598.

[41] Y. Koyama, I. Tanaka, H. Adachi, Y. Makimura, and T. Ohzuku, Journal of Power Sources. 119-121 (2003) 644-648.

[42] K.-Y. Oh, and B. I. Epureanu, Journal of Power Sources. 303 (2016) 86-96.

[43] Y. Qi, H. Guo, L. G. Hector, Jr., and A. Timmons, Journal of the Electrochemical Society. 157 (5) (2010) A558-A566.

[44] R. Yazami, and Y. Reynier, Journal of Power Sources. 153 (2006) 312-318.

[45] J. Wang, J. Purewal, P. Liu, J. Hicks-Garner, S. Soukazian, E. Sherman, A. Sorenson, L. Vu, H. Tataria, M. W. Verbrugge, Journal of Power Sources. 269 (2014) 937-948.

[46] D. Andrea, Battery Management Systems for Large Lithium-ion Battery Packs, Artech House, 2010.

[47] H. Teng, Y. Ma, K. Yeow, M. Thelliez, SAE International Journal of Passenger Cars Mechanical Systems. 4 (3) (2011) 1343-1357.

[48] F. He, D. Ewing, J. Finn, J. Wagner, L. Ma, SAE International Journal of Alternative Powertrains. 2 (1) (2013) 174-203.

[49] T. M. Bandhauer, S. Garimella, and T. F. Fuller, Journal of the Electrochemical Society. 158 (3) (2011) R1-R25.

[50] Z. Rao, S. Wang, Renewable and Sustainable Energy Reviews. 15 (2011) 4554-4571. 
[51] K. Mukai, Y. Kishida, H. Nozaki, and K. Dohmae, Journal of Power Sources. 224 (2013) 230-235.

[52] K.-Y. Oh, B. I. Epureanu, J. B. Siegel, and A. G. Stefanopoulou, Journal of Power Sources. 310 (2016) 118-129.

[53] J. Sugiyama, T. Tamura, and H. Yamauchi, Journal of Physics: Condensed Matter. 7 (1995) 9755-9764.

[54] V. A. Sethuraman, M. J. Chon, M. Shimshak, N. Van Windle, and P. R. Fuduru, Electrochemistry Communications. 12 (2010) 1614-1617.

[55] Y. Qi, L. G. Hector, C. James, and K. J. Kim, Journal of the Electrochemical Society. 161 (11) (2014) F3010-F3018.

[56] N. Lagakos, J. Jarzynski, J. H. Cole, and J. A. Bucaro, Journal of Applied Physics. 59 (12) (1986) 4017-4031.

[57] O. Olabisi and R. Simha, Macromolecules 8 (2) (1975) 206-210.

[58] D. J. Lacks and G. C. Rutledge, The Journal of Physical Chemistry. 98 (4) (1994) $1222-$ 1231.

[59] S. A. Jawad, G. A. J. Orchard, and I. M. Ward, Polymer. 27 (1986) 1201-1210.

[60] J. B. Nelson, and D. P. Riley, Proceedings of the Physical Society. 57 (6) (1945) 477.

[61] S. Mohan, Y. Kim, and A. G. Stefanopoulou, 2015 ASME Dynamic Systems and Control Conference. Columbus, USA (2015).

[62] N. A. Samad, Y. Kim, J. B. Siegel, and A. G. Stefanopoulou, 2015 IEEE Vehicular Power and Propulsion Conference. Montreal, Canada (2015) 1-6. 
[63] Y. Kim, N. A. Samad, K.-Y. Oh, J. B. Siegel, B. I. Epureanu, A. G. Stefanopoulou, 2016 American Control Conference. Boston, USA (2016). 